\title{
Series-elastic properties of strips of smooth muscle from pig urinary bladder
}

\section{R. van Mastrigt}

Department of Urology, Erasmus University Rotterdam, P.O. Box 1738, 3000 DR Rotterdam, The Netherlands

\section{E. A. Tauecchio}

Department of Biological \& Medical Physics, Erasmus University Rotterdarn, P.O. Box 1738, 3000 DR Rotterdam, The Netherlands

\begin{abstract}
The series elasticity of strips of smooth muscle from pig urinary bladder was investigated by means of a series of computerised quick-release and quick-stretch measurements with and without stimulation of the muscle, and at different shortenings and force levels. The results cannot be interpreted in terms of a discrete passive series-elastic element. They can, however, be interpreted in terms of the sliding-filaments model for contracting muscle.
\end{abstract} Keywords-Quick-release technique, Series elasticity, Sliding filaments model, Smooth muscle, Urinary
bladder

List of symbols

$a=$ constant factor representing geometry of muscle

$A=$ coefficient of exponential crossbridge elasticity

$\beta=$ exponent of exponential crossbridge elasticity

$\beta / l_{\mathrm{o}}=$ exponent in elastic characteristic of discrete elastic element

$\Delta F_{\mathrm{s}^{-}}=$corrected force decrease during stimulation and quick release (in the rising phase of the contraction)

$\Delta F_{\mathrm{s}+}=$ corrected force increase during stimulation and quick stretch (in the rising phase of the contraction)

$\Delta F_{o_{-}}=$corrected force decrease after stimulation and during quick release (in the falling phase of the contraction)

$\Delta F_{o+}=$ corrected force increase after stimulation and during quick stretch (in the falling phase of the contraction)

$\Delta l=$ extent of quick release or stretch

$\Delta x=$ elongation of series elastic element

$E_{o} / l_{o}=$ coefficient in elastic characteristic of discrete elastic element

$F=$ force

$F_{\Delta l}=$ force measured during a quick release or quick stretch

$F_{i s o}=$ maximum active isometric force during contraction

First received 25th November 1980 and in final form 13 th November 1981

$0140-0118 / 82 / 050585+10 \$ 01 \cdot 50 / 0$

(C) IFMBE: 1982
$F_{\text {trig }}=$ preset force-trigger level

$g(X)=$ force as a function of length of a crossbridge

$h=$ upper limit of crossbridge lengths

$l=$ length of muscle strip

$n_{o}(t)=$ number of active crossbridges during an isometric contraction

$n(X)=$ spectrum of lengths of crossbridges

$V_{t}=$ tissue volume of strip

$X=$ length of crossbridge

$x=$ macroscopic equivalent of $X$ obtained by addition of lengths of crossbridges which are in series

$\sigma_{\Delta i}=$ force measured during a quick release or quick stretch, normalised by dividing by the cross-sectional area of the strip

\section{Introduction}

THE series-elastic properties of muscle represent the elastic behaviour that can be measured by stretching or releasing a muscle during a contraction. This concept of series elasticity was first introduced by HILL (1938), for striated muscle. However, the model was very often found to be applicable to smooth muscle too; see e.g. Gordon and SIEGMAN (1971), Hellstrand (1979) and the review by MURPHY (1976). In this model for muscular behaviour, the development of active force in a muscle is described in terms of a contractile element (CE) in series with an elastic element (SE). During isometric contraction, the contractile element stretches the series-elastic element, thus developing force. It has been shown (VAN MASTRIGT et al., 1978b) that this model can be used to calculate fundamental properties of the contractile element (in particular the force-velocity relation 
describing this element) from an isometric contraction.

To do this we need a mathematical description of the series-elastic element. Åberg (1967), ALEXANDER (1976), Parmley and Sonnenblick (1967), Meiss (1978) and HALPERN et al. (1978) describe the series elasticity of smooth muscle as approximately exponential. If we interpret this in terms of an elastic element exerting a stress which depends exponentially on strain, the calculation becomes very simple (vAN DUYL et al., 1978), but results in an infinitely high maximum contraction velocity of the muscle, which is unrealistic (VAN MASTRIGT and GRIFFITHS, 1979a). An interpretation in terms of an element having an exponential elastic modulus yields more realistic results (VAN MASTRIGT and GRIFFITHS, 1979a) and is also in accordance with measurements of the passive properties of urinary bladder strips (VAN MASTRIGT et al., 1978a). Other authors like BLANGÉ et al. (1972), CHAPMAN and HARROWER (1977) and JULIAN et al. (1978), however, present measurements indicating that the series elasticity of muscle cannot be described in terms of a discrete passive series element, but depends on the activation of the muscle, or on the force the muscle exerts (BRESSLER and CLINCH, 1974; FORD et al., 1977; HalPERn and MUlvany, 1976). This is in agreement with the sliding-filaments model for contracting muscle, introduced by HUXLEY (1957) for modelling striated muscle, if the series elasticity is assumed to be located in the crossbridges.

Although the muscle structure on which the slidingflaments model is based cannot be seen in smooth muscle, the model is very often also applied to, or found to be applicable to, this type of muscle (BUTLER et al. 1976; Gordon and SIEGMAN, 1971; HUXLEY, 1957). However, many authors consider that the series elasticity in smooth muscle cannot be ascribed solely to the crossbridges but must be found at least partially external to them (Grood and MATES, 1975; SIEGMAN et al., 1976; HALPERN et al,, 1978; MEISS, 1978; HELLSTRAND and JOHANSSON, 1979). In view of the possible clinical value of a method of determining contractile properties from isometric contractions of the urinary bladder (VAN MASTRIGT et al., 1979b) the present study was undertaken to investigate the possibility of quantitative modelling of the series elasticity of urinary-bladder smooth muscle in terms of a discrete, passive elastic element.

Measurements were performed by means of the quick-release technique, because this method tests the series elasticity directly.

\section{Methods}

Experiments were performed on strips of pig urinary bladder wall measuring approximately $10 \times 27 \mathrm{~mm}$. The bladders were obtained from the local slaughterhouse. The strips were submerged in a physiological solution at a temperature of $37^{\circ} \mathrm{C}$. Contractions were evoked by electrical stimulation.

Four insulated silver wires were inserted into the strips, parallel to the short side, and a mass electrode was placed into the immersion fluid. Rectangular pulses of $30 \mathrm{~V}$, lasting $7 \mathrm{~ms}$ and at a repetition rate of $20 \mathrm{~Hz}$ (GrifFITHS et al., 1979), were applied. One of

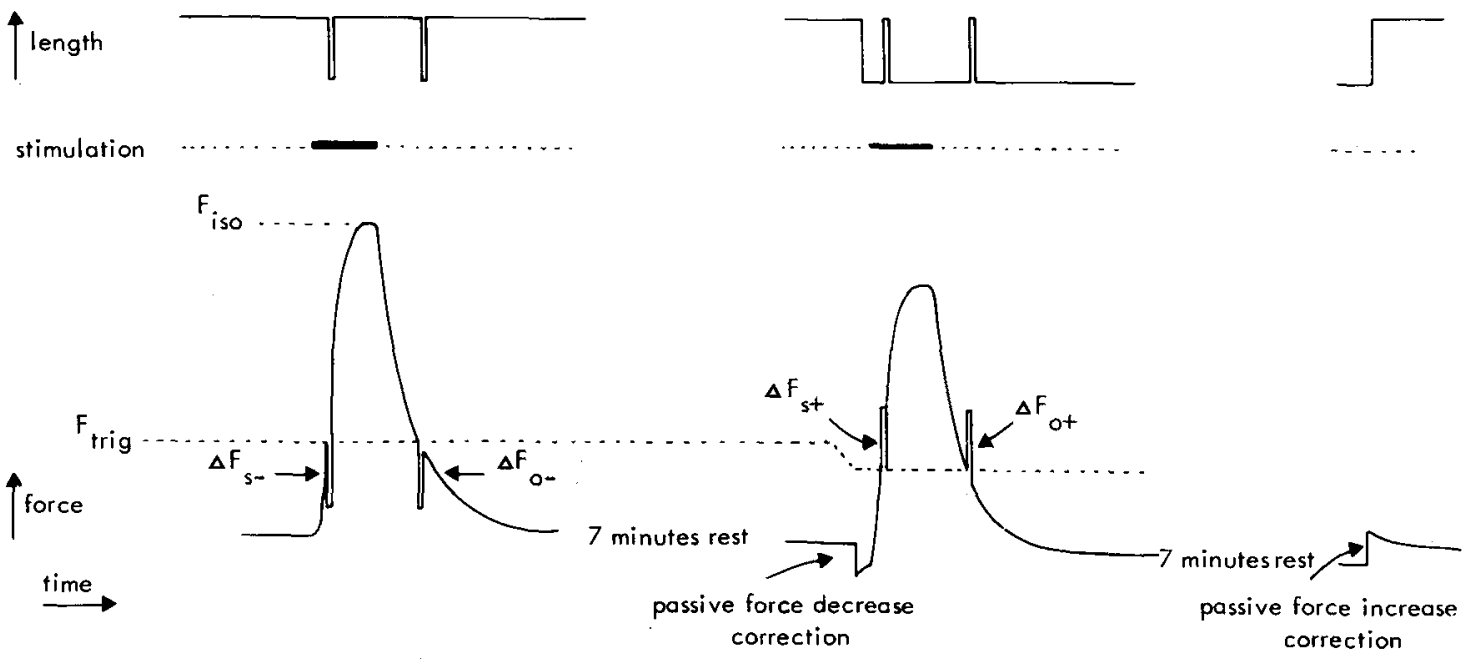

Fig. 1 Sequence of events during one measurement, comprising two contractions. Electrical stimulation of the muscle is indicated by a solid line, and stops as soon as force starts to decrease. During each contraction length changes are imposed during the rising and falling phases of the contraction, i.e. decreases in length during the first contraction and increases in length during the second. The resulting force changes are corrected for passive force changes by subtracting the response to an identical length change performed after the contractions. The symbol $\Delta F$ will be used for the force changes corrected in this way. 
the short sides of the strip was connected to a Grass FT 03C force transducer via a clamp with penetrating pins. The force signal obtained was fed to a minicomputer. An identical clamp on the other short side of the strip could be moved by a pneumatic cylinder, thus releasing or stretching the muscle stepwise by up to $50 \mathrm{~mm}$. Both the initial length and the amplitude of the length change of the muscle could be adjusted with micrometers. The electrical stimulation and the movement of the pneumatic cylinder were controlled by the minicomputer.

The strips were adjusted to an initial length where they were just taut. Prior to each stimulation, the force value was considered to be due to passive, parallel elastic properties of the strip, and was subtracted from all forces measured during contraction. One measurement consisted of the following sequence (see Fig. 1): Stimulation is turned on; when a preset trigger level (denoted by $F_{\text {trig }}$ ) is reached, the muscle strip is released for $250 \mathrm{~ms}$ and then reset to the original length. Next, stimulation is turned off as soon as the force starts to decrease, indicated by $F_{i s o}$ in Fig. 1. When the decreasing force again reaches the trigger level, a second quick release is performed. After a resting period of $7 \mathrm{~min}$, a control measurement is carried out, starting with a release. After the stimulation has been turned on, two quick stretches are measured in the same way as during the first stimulation, and, finally, the muscle is reset to the original length.

All releases and stretches during one measurement sequence imply the same preset amount of shortening or elongation. A typical force/time recording for one release pulse prolonged to $500 \mathrm{~ms}$ is shown in Fig. 2 . During each release or stretch pulse, 10 force samples are read by the computer at intervals of $25 \mathrm{~ms}$. The minimum or maximum in this sequence was taken to be representative of the force during the release or stretch.

The force change thus measured is assumed to be composed of a change in active force and a change in passive force. The latter force is due to the stretching of passive viscoelastic structures (VAN MASTRIGT et al., 1978a). Because active and passive force are approximately additive (GRIFFITHS et al., 1979), we can correct for the passive force changes by subtracting from the measured force change the response to an

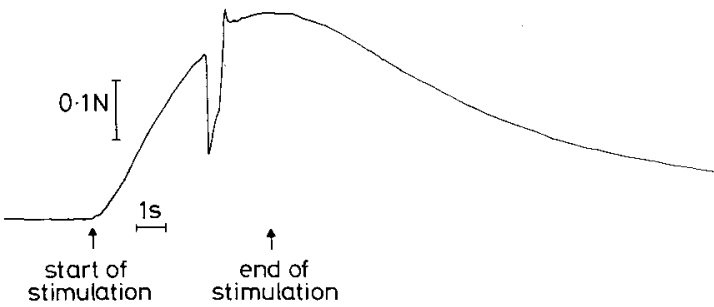

Fig. 2 Example of recorded force signal with one prolonged quick release and reset during contraction identical length change performed without stimulation of the muscle. These passive force changes are measured at the beginning and the end of the control measurement, as shown in Fig. 1. Thus, each measurement yielded four corrected values in the rising and falling phase of a contraction, and during release and stretch, denoted by $\Delta F_{s_{-}}, \Delta F_{o_{-}}, \Delta F_{s_{+}}, \Delta F_{o+}$, respectively. The maximum isometric force $F_{i s o}$ which the strip yielded usually decreased during the course of the measurements. The first few contractions measured on each strip yielded an $F_{i s o}$ between 0.8 and $1.6 \mathrm{~N}$. This yields an isometric active stress between $1 \times 10^{4}$ and $2 \times 10^{4} \mathrm{~N} \mathrm{~m}^{-2}$.

This is low compared with values found for other smooth muscles $\left[2.5-35 \times 10^{4} \mathrm{~N} \mathrm{~m}^{-2}\right.$ (HALPERN et al., 1978; Hellstrand, 1979; MURPHY, 1976)], but it correlates well with the maximum pressures measured in the normal urinary bladder (VAN MASTRIGT and GRIFFITHS, 1979a).

Measurements were continued until $F_{i s o}$ was lower than $F_{\text {trig }}$. Generally 10 to 20 contractions could be measured on one strip. This rapid deterioration is at least partly due to the fact that quick stretches were also applied, which probably damaged the strip (FORD et al., 1977). However, a maximum possible number of about 20 contractions has been found before for strips prepared in this way, even without quick stretches (GRIFFITHS et al., 1979). The release and stretch speeds of the pneumatic device are in the order of $200 \mathrm{~mm} \mathrm{~s}^{-1}$. This is well above the maximum speed of contraction, which was estimated at $5 \mathrm{~mm} \mathrm{~s}^{-1}$ for these strips (GRIFFITHS et al., 1979; VAN MASTRIGT and GRIFFITHS, 1979a). This $v_{\max }$ (normalised 0.2 strip lengths per second which agrees very well with values found for other smooth muscles (MURPHY, 1976)) is a factor of 20-100 lower than values found for striated muscle (MurPhy, 1976). This justifies the use of a relatively slow force transducer and low sampling rate.

\section{Results of measurements with steps of equal length and constant trigger level}

We consider the four measured $\Delta F \mathrm{~S}$ as the dependent variables in our experiment. There are three

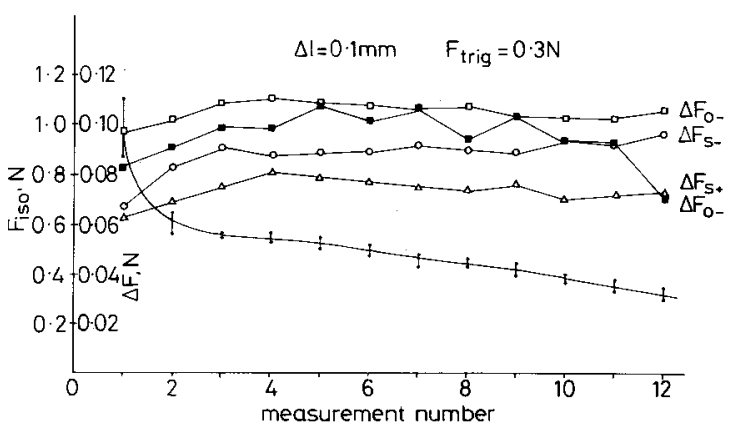

Fig. 3 Corrected force decrease and increase steps measured in experiment during which only $F_{\text {iso }}$ varied 
independent variables; $F_{i s o}, \Delta l$ and $F_{i r i g}$. In this Section we describe the relation between the $\Delta F$ s and $F_{i s o}$. The latter varies considerably during each experiment. Measurements were performed on five strips. $F_{\text {trig }}$ was fixed at $0.3 \mathrm{~N}$ and $\Delta l$ was $0.1 \mathrm{~mm}$ for two strips and $0.3 \mathrm{~mm}$ for the other three. The results of one measurement are shown in Fig. 3. Two values of $F_{i s o}$ were obtained for each measurement. The higher plotted value was always measured in the first of the two contractions.

Although some kind of common pattern can be seen in the $\Delta F$ and $F_{\text {iso }}$ curves, the dependence is so slight that, to a first approximation, we can regard the $\Delta F \mathrm{~s}$ as independent of $F_{\text {iso }}$. The averages and standard

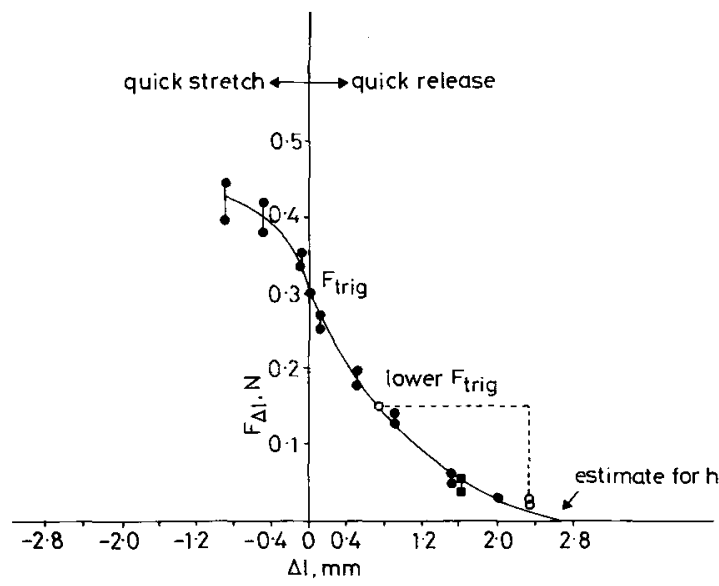

Fig. 4 Active force as a function of quick shortening or stretch. Dotted line shows how quick releases measured at a lower $F_{\text {trig }}$ value should be plotted. Starting from a point on the curve corresponding to the lower $F_{\text {trig }}$ (open circle), the length change during the quick release is plotted horizontally and the resulting force change is plotted vertically, yielding the two open circles (which do not fit the curve). The solid squares represent the same data corrected for the lower $F_{\text {trig }}$ value by a factor $F_{\text {trig } 2} / F_{\text {trig } 1}$, as explained in Section 5 deviations for all measurements are shown in Table 1. A significant (student's $t$ test, 95\%), though rather small, difference between the four $\Delta F$ s is always seen. The $\Delta F$ s measured without stimulation (i.e. in the falling phase of the contraction) are $18 \%$ greater on the average than those measured with stimulation (in the rising phase of contraction). This holds good for quickrelease as well as for quick-stretch measurements. Furthermore, when we omit the signs, the responses to quick stretches are $14 \%$ smaller on the average than those to quick releases. This will be discussed in the following Section. Apart from these second-order effects, we conclude that, to a first approximation, the measured decrease or increase in force is independent of $F_{i s o}$.

\section{Results of measurements with steps of varying length}

Next, we investigated the dependence of the $\Delta F \mathrm{~s}$ on $\Delta l$ at a constant $F_{\text {trig }}$ of $0 \cdot 3 \mathrm{~N}$. Eleven experiments were performed. A typical plot of the active force $F_{\Delta l}$ exerted by the strip during shortening or elongation, as a function of $\Delta l$, is shown in Fig. 4. $F_{\Delta l}$ is calculated from the $\Delta F$ s by adding to $F_{\text {trig }}$ with the appropriate sense, as follows:

$$
\begin{aligned}
& F_{\Delta l}=F_{\text {trig }}-\Delta F_{s-} \quad \text { and } \quad F_{\Delta l}=F_{\text {trig }}-\Delta F_{o-} \\
& \text { for } \Delta l>0 \quad \text { (shortening) } \\
& F_{\Delta l}=F_{t r i g}+\Delta F_{s+} \quad \text { and } \quad F_{\Delta l}=F_{t r i g}+\Delta F_{o+} \\
& \text { for } \Delta l<0 \quad \text { (elongation) }
\end{aligned}
$$

Thus, for each value of $\Delta l$, two force values are plotted, the upper one representing the force change measured in the falling phase of a contraction and the lower one the force change measured in the rising phase of the contraction. As can be seen, the results obtained with quick releases and quick stretches are consistent in that they yield a continuous curve. The decrease in the slope of the curve for high $\Delta l$ can be understood from the fact that the force during the quick stretch then exceeds the maximum force $F_{i s o}$, which causes the

Table 1. Averages and relative standard deviations of measurements made on six strips with constant $\Delta$ land constant $F_{\text {triq }}(0 \cdot 3 \mathrm{~N})$, so that only $F_{\text {iso }}$ varied

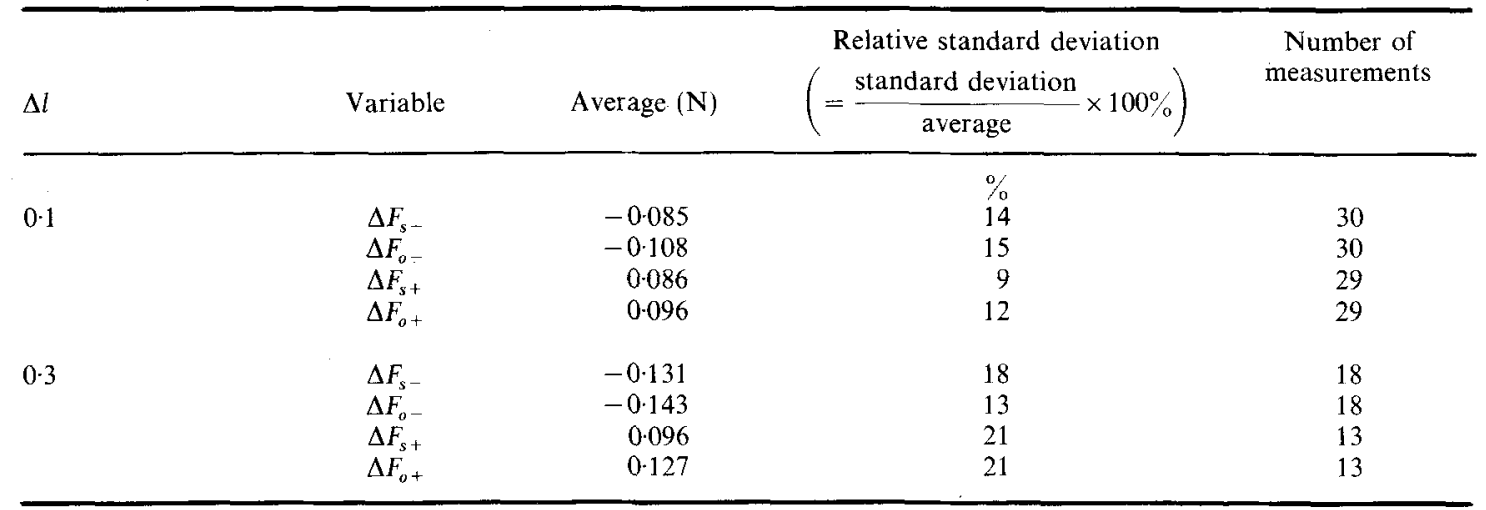


contractile 'machinery' to 'slip' (HILL, 1938; LEVIN and WYMAN, 1927; JULIAN et al., 1978).

The curve shown in Fig. 4 can be understood as the elastic characteristic of a discrete passive series-elastic element. We plotted the 'stiffness' of this hypothetical elastic element (the derivative $d F_{\Delta l} / d \Delta l$ normalised with respect to the cross-sectional area of the strip to yield $d \sigma_{\Delta l} / d \Delta l$ ) as a function of $\sigma$ in Fig. 5. The results of

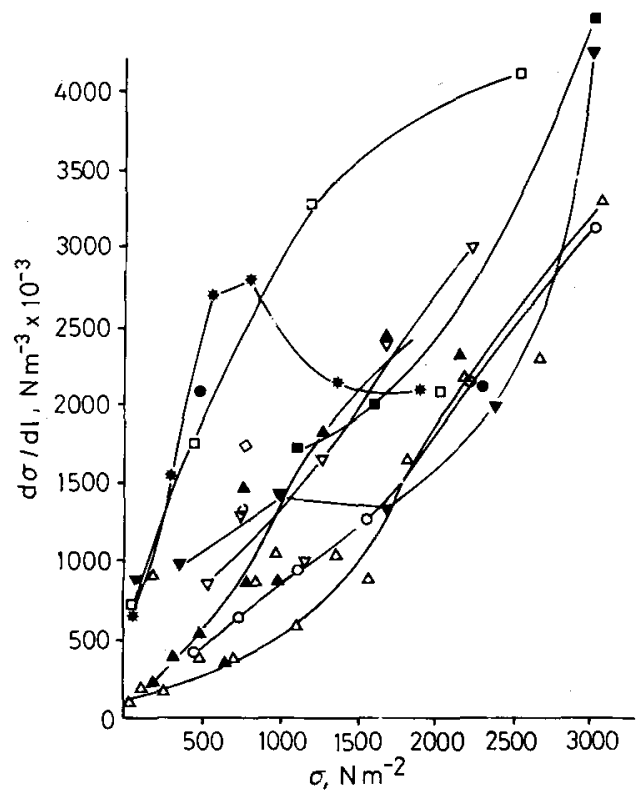

Fig. 5 Stiffness of hypothetical series-elastic element as a function of active stress exerted. The stress was calculated by dividing the force during a quick release by the cross-sectional area of a strip. The stiffness is the derivative of this stress with respect to the amplitude of the quick release

$\begin{array}{ll}\star \text { curve } 2004 & \nabla \text { curve } 2010 \\ \nabla \text { curve } 2005 & \square \text { curve } 2011 \\ \text { curve } 2006 & \square \text { curve } 2012 \\ \triangle \text { curve 2009 } & \text { A curve 2013 }\end{array}$

the best eight out of the 11 measured curves are displayed. As the plotted curves are not straight lines, the measured quick-release curves (Fig. 4) are not directly exponential (see Introduction). It was concluded from measurements performed on whole bladders (VAN MASTRIGT and GRIFFITHS, 1979a) and bladder-wall strips (VAN MASTRIGT et al., 1978a) that the series elasticity of urinary bladder-wall muscle can be adequately described by the function

$$
F=\frac{V_{t}}{l} \frac{E_{o}}{l_{o}} \Delta x \exp \left(\frac{\beta}{l_{o}} \Delta x\right)
$$

where

$$
\frac{\beta}{l_{o}} \text { and } \frac{E_{o}}{l_{o}} \text { are parameters }
$$

$V_{t}$ is the tissue volume of the strip

$l$ is the length of the strip

and $\Delta x$ is the elongation of the series-elastic element.

This formula can be fitted to the measured $F_{\Delta l}$ curves by putting $\Delta x=h-\Delta l$, where $h$ is the value of $\Delta l$ when $F_{\Delta l}=0$. The fitting was performed by estimating $h$, at $\mathrm{F}_{\Delta l}=0$, by estimating $h$ from the $F_{\Delta l}$ against $\Delta l$ plot (see Fig. 4), dividing $F_{\Delta l}$ by $(h-\Delta l)$ and plotting the result semilogarithmically. Nine out of the 11 curves measured could be described in this way.

Table 2. Averages and standard deviations of parameters of the hypothetical, discrete, passive series-elastic element, derived from the data of Fig. 5

\begin{tabular}{lccc}
\hline Parameter & Average & $\begin{array}{c}\text { Relative } \\
\text { standard } \\
\text { deviation }\end{array}$ & $\begin{array}{c}\text { Number of } \\
\text { measurements }\end{array}$ \\
\hline & $\%$ & \\
$\beta / l_{o}$ & $674\left(\mathrm{~m}^{-1}\right)$ & 31 & 9 \\
$E_{o} / l_{o}$ & $475 \times 10^{3} \cdot \mathrm{Nm}^{-3}$ & 70 & 9 \\
\hline
\end{tabular}

Averages and standard deviations of the resulting parameters are shown in Table 2 . The average values can be compared with those measured on a whole bladder (VAN MASTRIGT and GRIFFITHS, 1979a) by applying a correction factor for the different geometry (VAN MASTRIGT, unpublished data). The high standard deviations found in Table 2 show that this modelling of the series elasticity of the urinary bladder wall is not very successful.

At the end of an experiment, when $F_{\text {iso }}$ became lower than $F_{\text {trig }}$, one or more measurements were sometimes performed at a lower value of $F_{t r i q}$. In the case of Fig. 4, such a measurement was performed with $F_{\text {trig }}=0.15 \mathrm{~N}$. On the assumption of a discrete, passive series-elastic element, such a measured point should be represented by the open circles shown in Fig. 4. Starting from the point along the curve corresponding with the lower value of $F_{\text {trig }}$ (open circle), the amplitude of the length change during the quick release is plotted horizontally and the resulting force change vertically, yielding the two open circles seen near the foot of the curve. This was tried in five experiments but the extra measurements never fitted the original curve.

A better way of interpreting these quick-release data will be presented in Section 6.

\section{Results of measurements with varying trigger forces}

Finally, the dependence of the $\Delta F$ s on $F_{\text {trig }}$ was investigated. Measurements were performed on five strips. For two strips, $\Delta l$ was fixed at $0.2 \mathrm{~mm}$; the other three were measured with $\Delta l=1 \mathrm{~mm}$. The resulting 
$\Delta F \mathrm{~s}$, which were divided by $\Delta l$ to yield stiffnesses, were found to be directly proportional to $F_{\text {trig. }}$. This is illustrated in Fig. 6. The quick-stretch measurements here were all performed at the same $F_{\text {trig }}$, to see whether this increased the reproducibility. This, however, was not the case, as can be seen in Table 3. The proportionality found between force change and $F_{t r i g}$ necessitates a number of remarks.

First, we now have a method for correcting

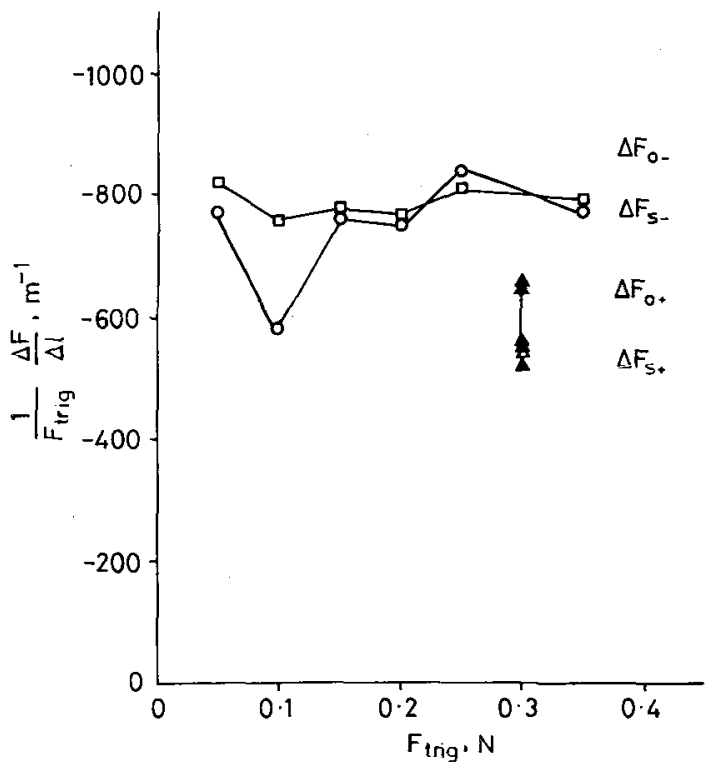

Fig. 6 Results of measurements on one strip made at constant $\Delta$ l. $F_{\text {trig }}$ is kept constant for the quick-stretch measurements, and is varied for the quick-release measurements. The results show that the quotient $1 / F_{\text {trig }} \frac{\Delta F}{\Delta l}$ does not depend on $F_{\text {trig }}$ measurements made at different $\dot{F}_{\text {trig }}$ levels, as presented in Section 4. For instance, the measurement represented in Fig. 4 by open circles does fit (solid squares) when corrected for the lower $F_{\text {trig }}$ value by the simple factor $F_{\text {sriq } 2} / F_{\text {trig } 1}$.

In four out of the five experiments from Section 4 in which measurements at lower $F_{\text {trig }}$ values were available, these could be fitted to the measured curves by applying this correction factor. Secondly the observation that the measured force change is proportional to the force level at which the length change is imposed has been made by many authors (BRESSLER and ClINCH, 1974; FORD et al., 1977; Julian et al., 1978; MeIss, 1978; Hellstrand, 1979). Most of them conclude that this means that the force/length characteristic is exponential. We found this not to be the case (see Section 4). Furthermore, the proportionality was also found for large length changes $(\Delta l=1 \mathrm{~mm}$, which is $38 \%$ of $h$, the length change for which $F_{\Delta l}=0$ ), which, in terms of a passive discrete series elasticity, can only be understood from a linear force/length characteristic parallel to the force axis.

Finally, these findings have to be compared with those of Section 4. If we model the series elasticity of this type of smooth muscle by a discrete, passive series element, this comparison can be made in terms of stiffness, as explained in Fig. 7.

The stiffness data of Fig. 5 are replotted in Fig. 8. Two straight lines have been inserted representing the measurements presented in this Section for $\Delta l=0.2 \mathrm{~mm}$. It can be seen that especially at high forces the stiffnesses measured in the two different ways do not agree. We must thus abandon the idea of one discrete passive series element representing the elasticity of urinary bladder smooth muscle, as has also been concluded by other authors for other muscle types (BLANGÉ et al., 1972; CHAPMAN and HARROWER, 1977).

Table 3. Results of measurements made with constant $\Delta l(\Delta l=1 \mathrm{~mm}$ for three strips and $\Delta l=0 \cdot 2 \mathrm{~mm}$ for two strips $)$ and varying or constant $F_{\text {trig }}$

\begin{tabular}{|c|c|c|c|c|c|}
\hline$\Delta l$ & $F_{\text {trig }}$ & Parameter & Average value & $\begin{array}{c}\text { Relative } \\
\text { standard deviation }\end{array}$ & $\begin{array}{l}\text { Number of } \\
\text { measurements }\end{array}$ \\
\hline \multirow{3}{*}{$\mathrm{mm}$} & \multirow{3}{*}{ variable } & & $\mathrm{m}^{-1}$ & $\%$ & \\
\hline & & $1 / F_{\text {trig }} \times \Delta F_{s}-/ \Delta l$ & 649 & 18 & 21 \\
\hline & & $1 / F_{\text {triq }} \times \Delta F_{o-} / \Delta l$ & 714 & 7 & 20 \\
\hline \multirow[t]{2}{*}{1} & \multirow[t]{2}{*}{ constant } & $\begin{array}{c}1 / F_{\text {trig }} \times \Delta F_{s+} / \Delta l \\
\left(F_{\text {triq }}=0.3 \mathrm{~N}\right)\end{array}$ & 490 & 18 & 17 \\
\hline & & $\begin{array}{c}1 / F_{t r i g} \times \Delta F_{o+} / \Delta l \\
\left(F_{t r i g}=0.3 \mathrm{~N}\right)\end{array}$ & 611 & 23 & 17 \\
\hline \multirow[t]{4}{*}{$0 \cdot 2$} & \multirow[t]{2}{*}{ variable } & $1 / F_{\text {trig }} \times \Delta F_{s-} / \Delta l$ & 529 & 44 & 12 \\
\hline & & $1 / F_{\text {trig }} \times \Delta F_{0-} / \Delta l$ & 876 & 6 & 12 \\
\hline & \multirow[t]{2}{*}{ constant } & $1 / F_{\text {irig }} \times \Delta F_{s+} / \Delta l$ & 789 & 19 & 14 \\
\hline & & $1 / F_{\text {trig }} \times \Delta F_{o+} / \Delta l$ & 795 & 6 & 14 \\
\hline
\end{tabular}


6 Interpretation of results in terms of sliding filaments model

In the preceding Sections we described three findings which cannot be interpreted in terms of the model of HILL (1938) using a discrete passive series elastic element, namely

(i) the proportionality of stiffness and force for large length changes (Section 5, Table 3)

(ii) the differences in stiffness found by varying $\Delta l$ and $F_{i r i g}$ (Fig. 8)

(iii) the fact that measurements made at a lower $F_{\text {trig }}$ (Section 4) must be corrected by a factor $F_{\text {trig } 2} / F_{\text {trig } 1}$ (Section 5)

We will now consider these findings in terms of the sliding-filaments model proposed by HUXLEY (1957, 1974). If we assume that there is no elasticity apart from that in the crossbridges (BLANGÉ et al., 1972; HUXLEY, 1957) the force the muscle exerts at any moment can be expressed as the integral of the forces exerted by the crossbridges over a spectrum of crossbridge lengths (HUXLEY, 1957):

$$
F=a \int_{-\infty}^{+\infty} n(X) g(X) d X
$$

where

$$
\begin{aligned}
F & =\text { force exerted by muscle } \\
X & =\text { length of crossbridge } \\
n(X) & =\text { spectrum of lengths of crossbridges } \\
g(X) & =\text { force as function of length of crossbridge } \\
a & =\text { constant factor representing geometry etc. }
\end{aligned}
$$

A real muscle, or muscle strip consists of a lot of contractile units (sarcomeres) in series, taken into account by replacing the parameter $X$ by $x$, its macroscopic equivalent. It is assumed that in the isometric case the crossbridge length is always distributed uniformly within a certain range (HUXLEY, 1957):

$$
\begin{aligned}
& n(x)=0 \text { for } x>h \text { and } x<0 \\
& n(x)=n_{a} \text { for } 0 \leqslant x \leqslant h .
\end{aligned}
$$

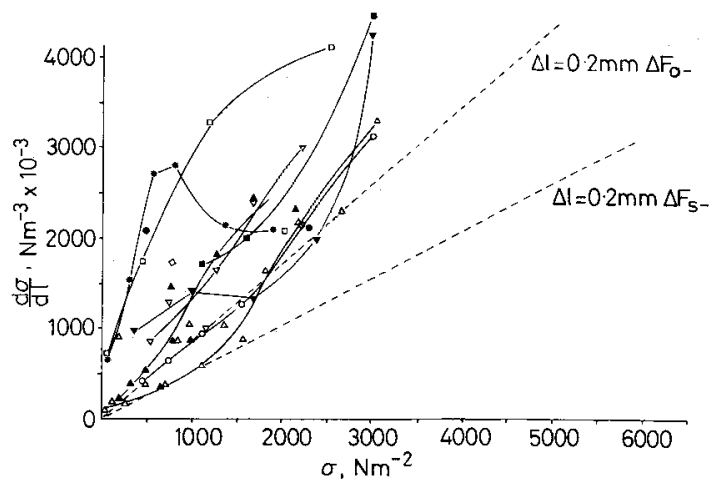

Fig. 8. The stiffness data of Fig. 5 (measured at constant trigger level and varying stretch or release length) compared with the two straight lines representing the stiffness measured as a function of $F_{\text {trig }}$ at a constant value of $\Delta l$ (Section 5). It will be seen that the agreement between the results obtained by these two methods is poor, especially at high forces

$\begin{array}{ll}\star \text { curve } 2004 & \nabla \text { curve } 2010 \\ \nabla \text { curve 2005 } & \square \text { curve 2011 } \\ \bigcirc \text { curve 2006 } & \square \text { curve 2012 } \\ \Delta \text { curve 2009 } & \text { curve 2013 }\end{array}$

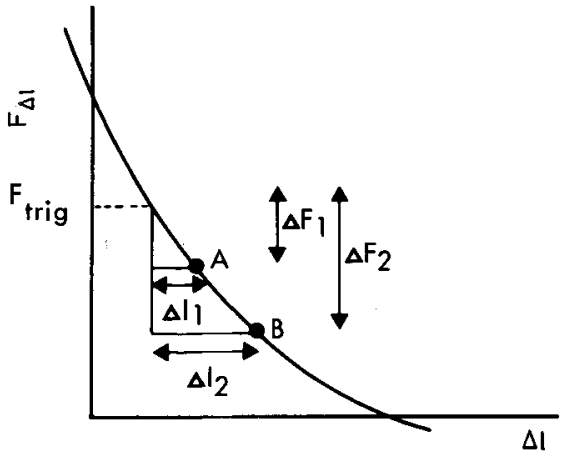

$a$

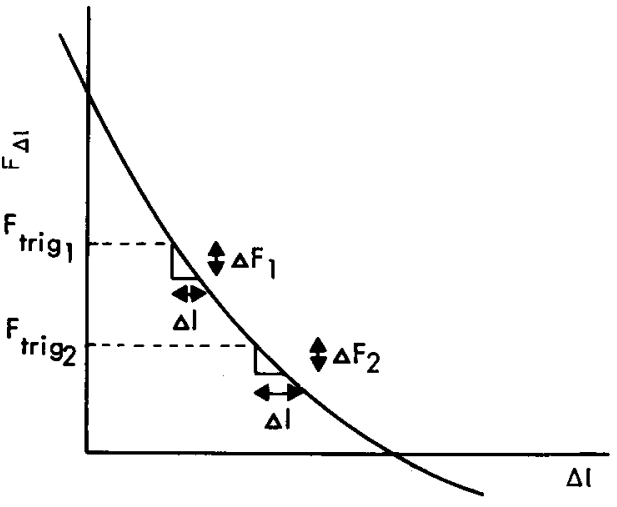

$b$

Fig. 7 Force changes measured as a function of $\Delta l(a)$ can be compared with force changes measured as a function of $F_{\text {trig }}(b)$ by comparing the stiffness of the hypothetical, discrete, passive series-elastic element at a given force in the two cases. In case (a), the stiffness $\left(=\right.$ the slope of the quick-release curve) can be approximated to by $\left(\Delta F_{2}-\Delta F_{1}\right) /\left(\Delta l_{2}-\Delta l_{1}\right)$. In case $(b)$, we can approximate to the stiffness directly by calculating $\Delta F / \Delta l$, provided $\Delta l$ is relatively small 
During an isometric contraction the build-up of force is due to the successive formation of crossbridges:

$$
F(t)=a n_{o}(t) \int_{0}^{h} g(x) d x
$$

What happens during a quick release depends on whether the crossbridges can exert a negative force or not. Some authors conclude that they cannot (BLANGÉ et al., 1972), though HUXLEY (1957) states that they can, because a quick-release curve approaches the $\Delta l$-axis sharply. This claim is not falsified by our data (it means that $d \sigma_{\Delta} / d \Delta l \neq 0$ for $\sigma_{\Delta l}=0$ in Fig. 5) but we believe that this can be understood by assuming crossbridges which do not exert negative force but do exert force at zero extension (cf. eqn. 10). We will thus assume that

$$
g(x)=0 \text { for } x<0 .
$$

During a quick release, the length distribution of the crossbridges shifts to the origin:

$$
F_{\Delta l}=a n_{0}(t) \int_{0}^{h-\Delta l} g(x) d x .
$$

At this stage of our argument, we can understand why the results of measurements at different forces (presented in Section 5) yielded a constant quotient of $\Delta F$ and $F_{\text {trig }}$. Since $F_{\text {trig }}$ is simply equal to $F(t)$ at a given $(t)$, we find:

$$
\begin{aligned}
\frac{1}{F_{\text {trig }}-\frac{\Delta F}{\Delta l}} & =\frac{1}{F_{\text {trig }}} \frac{F_{\text {trig }}-F_{\Delta l}}{\Delta l} \\
& =\frac{\int_{0}^{h} g(x) d x-\int_{0}^{h-\Delta l} g(x) d x}{\Delta l \int_{0}^{h} g(x) d x}
\end{aligned}
$$

which does not depend on $n_{o}(t)$ and is constant at a constant value of $\Delta l$. For a quantitative description of our results we have to determine $g(x)$. Eqn. 6 yields

$$
\frac{d F_{\Delta l}}{d \Delta l}=a n_{o}(t) \cdot-g(h-\Delta l)
$$

Fig. 9 shows a semi-logarithmic plot of this derivative against $\Delta l$ from seven out of the 11 quick-release curves measured, showing that they all fit the same straight line (apart from some marked outliers near $\Delta l=0$ ).
The other measured curves showed large variations in $d F_{\Delta l} / d \Delta l$. Thus

$$
\frac{d F_{\Delta l}}{d \Delta l}=-A e^{-\beta \Delta l}
$$

Combination of eqns. 8 and 9 yields

$$
\begin{aligned}
& g(x)=\frac{A}{a n_{o}(t)} e^{\beta(x-h)} . \\
& F_{\Delta l}=\frac{A}{\beta}\left[e^{-\beta \Delta l}-e^{-\beta h}\right] .
\end{aligned}
$$

Here $\frac{A}{\beta}$ is not a constant but will probably depend on $\dot{n}_{o}(t)$ in some way. The validity of eqn. 11 can be checked by estimating $h$ from a quick-release curve as the point where $F_{\Delta l}=0$ (see Fig. $4, h=2.6 \mathrm{~mm}$ ), next estimating $\frac{A}{\beta}$ from $F_{\text {trig }}$ (where $\Delta l=0$ ) by inserting the estimated $h$, and then plotting $\frac{F_{\Delta l}}{A / \beta}+e^{-\beta h}$ semilogarithmically as a function of $\Delta l$. This should (and does) yield a straight line.

As a result of these calculations based on the slidingfilaments model, and some rather restrictive assumptions, we see that the findings presented in the beginning of this Section can casily be explained. The proportionality of stiffness and force follows from eqn. 7. The differences in stiffness obtained by varying $\Delta l$ and $F_{\text {trig }}$ are explained by the fact that in the first type of

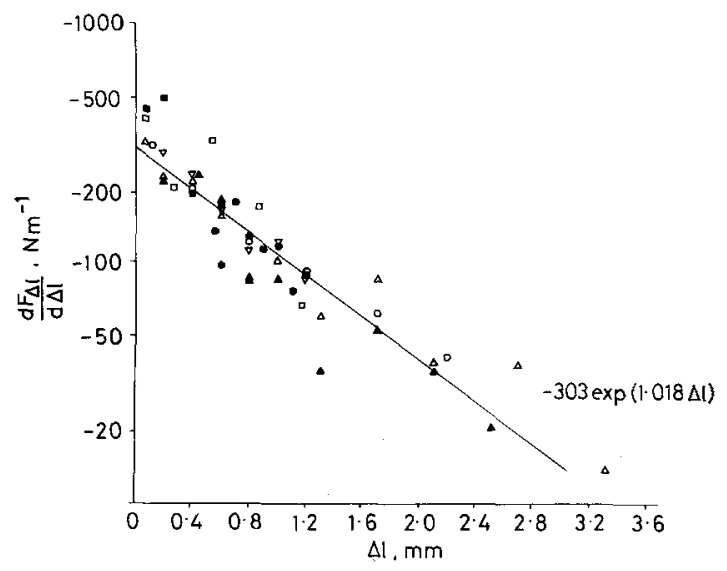

Fig. 9 Semilogarithmic plot of $d F_{\Delta /} / d \Delta l$ versus $\Delta l$, calculated from quick-release curves such as that of Fig. 4

$\begin{array}{ll}- \text { curve 2001 } & \square \text { curve 2011 } \\ \bigcirc \text { curve 2006 } & \square \text { curve 2012 } \\ \triangle \text { curve 2009 } & \mathbf{\Delta} \text { curve 2013 } \\ \nabla \text { curve 2010 } & \end{array}$


measurements eqn. 11 holds, and in the second case eqn. 7 .

Finally; measurements made at lower $F_{\text {trig }}$ values should be corrected according to eqn. 7, and not according to eqn. 11 .

\section{Conclusions and discussion}

The quick-release and quick-stretch measurements we performed cannot be described in terms of one discrete passive series elastic element. They can however be described in terms of the sliding-filaments theory, assuming that

(i) the series elasticity resides in the crossbridges

(ii) the crossbridges cannot push, only pull

(iii) the lengths of the crossbridges during an isometric contraction are distributed uniformly within a given range.

Especially for the first assumption, the parameter $h$ is of crucial importance. This is the value of $\Delta l$ which makes the force during a quick release zero, see eqn. 11. In the first place, this value is independent of the force level from which release starts. However large the contractile force is, it can always be made exactly zero by the same amount of release. This deduction is confirmed by the measurements of Section 5 , and is in complete contradiction to the predictions made on the basis of a discrete passive series elastic element. Secondly, the parameter $h$ varied from 1.2 to $5.5 \mathrm{~mm}$ in our measurements, with an average of $2.6 \mathrm{~mm}$. Since our average strip length amounted to $27 \mathrm{~mm}$, we see that a quick shortening by about $10 \%$ of the muscle length is necessary to remove all tension. This is a not uncommon value for smooth muscle (values found in literature range from 5 to $20 \%$, MURPHY (1976)). It is however very large in comparison with values found for striated muscle, which range from 0.4 to $2 \%$ (FORD et al., 1977; BRESSLER and CLINCH, 1974; BLANGÉ et al., 1972). Most authors therefore conclude that a large part of the series elasticity of smooth muscle must be found outside the corssbridges (HELlstrand, 1979; HALPERN et al., 1978; SIEGMAN et al., 1976; MURPHy, 1976). Perhaps a large part of the series elasticity does not reside in the crossbridges, but in a structure in series with a number of crossbridges. Series elasticity might for instance be found in the filaments. In this case the dependence of stiffness on force and the other observations mentioned in Section 6 can still be understood in much the same way. The orientation of cells within the tissue is also relevant in this connection. It is not certain whether these are in series or in parallel (MURPHY, 1976) although there is some evidence that cell length is proportional to total tissue length (Hellstrand, 1979), which implies that no gross reorientation of cells within the tissue takes place as a result of stress. It will be clear that more anatomical data is necessary.

Finally, we should mention a pertinent observation not covered by the theory presented. We noted that the force response both to quick releases and to quick stretches was always larger during the declining part of a contraction than during the rising part. The same effect was found by MeIss (1978) in rabbit mesotubarium smooth muscle. The reverse of this effect, i.e. a greater stiffness in the rising phase of contraction, was predicted by GROOD and MATES (1975) on the basis of a model with the series elasticity partially in the crossbridges and partially external to these. HeLlSTRAND and JOHNSTON (1979) found this reverse effect in the rabbit urinary bladder.

\section{References}

ÅberG, A. K. G. (1967) The series elasticity of active taenia coli in vitro. Acta Physiol. Scand., 69, 348-354.

ALEXANDER, R. S. (1976) Series elasticity of urinary bladder smooth muscle. Am. J. Physiol., 231-5, 1337-1342.

Blangé, T., Karemaker, J. M. and Kramer, A. E. J. L. (1972) Elasticity as an expression of cross-bridge activity in rat muscle. Pflïgers Arch., 336, 277-288.

Butler, T. M., SiEgman, M.J. and Davies, R. E. (1976) Rigor and resistance to stretch in vertebrate smooth muscle. $\mathrm{Am}$. J. Physiol., 231, 1509-1514.

Bressler, B. H. and CLINCH, N. F. (1974) The compliance of contracting skeletal muscle. J. Physiol, 237, 477-493.

Chapman, A. E., Harrower, P. T. (1977) Linear approximations of muscle mechanics in isometric contraction. Biol. Cybernetics, 27, 1-7.

Coolsaet, B. L. R. A., van Duyl, W. A., van Mastrigt, R., VAN DER ZWART, A. (1975) Visco-elastic properties of the bladder wall. Urologia Internationalis, 30, 16-26.

van DuYl, W. A., Coolsaet, B. L. R. A. and van Mastrigt, R. (1978) A new clinical parameter for the assessment of the contractility of the urinary bladder. Urologia Internationalis 33, 31-39.

Ford, L. E., HuXley, A. F. and Simmons, R. M. (1977) Tension responses to sudden length change in stimulated frog muscle fibres near slack length. J. Physiol., 269, 441 515.

Gordon, A. R. and Siegman, M. J. (1971) Mechanical properties of smooth muscle. Am. J. Physiol., 221, 12431254.

Griffiths, D. J., van Mastrigt, R., van DuYl, W. A. and CoOlsaET, B. L. R. A. (1979) Active mechanical properties of the smooth muscle of the urinary bladder. Med. \& Biol. Eng. \& Comput., 17, 281-290.

Grood, E. S. and MATES, R. E. (1975) Influence of crossbridge compliance on the force-velocity relation of muscle. Am. J. Phys., 228-1, 244-249.

Halpern, W. and Mulvany, M. J. (1976) Tension responses to small length changes of vascular smooth muscle cells. Am. J. Phys., 21P-23P.

Halpern, W., Mulvany, M. J. and Warshaw, D. M. (1978) Mechanical properties of smooth muscle cells in the walls of arterial resistance vessels. $J$. Physiol., 275, 85-101.

Ham, A. W. (1957) Histology. London, Pitman Medical Publishing Co. Ltd.

Hellstrand, P. (1979) Mechanical and metabolic properties related to contraction in smooth muscle. Acta Phys. Scand. Suppl., 464.

Hellstrand, P., Johansson, B. (1979) Analysis of the length response to a force step in smooth muscle from rabbit urinary bladder. Acta Physiol. Scand., 106, 221-238. 
HiLl, A. V. (1938) The heat of shortening and the dynamic constants of muscle. Proc. Roy. Soc. of London B, 126, 136195.

Huxley, A. F. (1957) Muscle structure and theories of contraction. Progress in Biophysics and Chemistry, 7, 255318 .

HuXley, A. F. (1974) Review Lecture. Muscular Contraction. J. Physiol., 243-1, 1-43.

Julian, F. J., Moss, R. L. and Sollins, M. R. (1978) The mechanism for vertebrate striated muscle contraction. Circ. Res., 42-1, 2-14.

Levin, A. and WYMAN, J. (1927) The viscous elastic properties of muscle. Proc. Roy. Soc. of London, B, 101, $218-243$.

van Mastrigt, R., Coolsaet, B. L.: R. A. and van Duyl, W. A. (1978a) Passive properties of the urinary bladder in the collection phase. Med. \& Biol. Eng. \& Comput., 16, 471482.

van Mastrigt, R., Griffiths, D. J. and van Duyl, W. A. $(1978 b)$ The assessment of the contractility of the urinary bladder. Preprints VIIIth International Continence
Society Meeting, Pergamon Press.

VAN Mastrigt, R. and Griffiths, D. J. (1979a) The contractility of the urinary bladder. Urologia Internationalis, 34, 410-420.

van Mastrigt, R., Coolsaet, B. L. R. A. and van DUyL, W. A. (1979b) Clinical determination of the maximal contraction velocity of the urinary bladder. Proceedings IXth annual meeting International Continence Society, Guido Guidotti Editore, Rome.

MeIss, R. A. (1978) Dynamic stiffness of rabbit mesotubarium smooth muscle: effect of isometric length. Am. J. Physiol., 234, C14-C26.

Murphy, R. A. (1976) Contractile system function in mammalian smooth muscle. Blood vessels, 13, 1-23.

Parmley, W. W. and Sonnenblick, E. H. (1967) Series elasticity in heart muscle. Circ. Res. $X X, 112-123$.

Siegman, M. J., Butler, T. M., Mooers, S. U. and Davies, R. E. (1976) Calcium dependent resistance to stretch and stress relaxation in resting smooth muscle. Am. J. Phvs., 231-5, 1501-1508. 